\title{
Testing the spectral resolutions of the new multispectral sensors for detecting Phaeosphaeria leaf spot (PLS) infestations in maize crop
}

\author{
${ }^{1}$ John Odindi, Odindi@ukzn.ac.za, ${ }^{2}$ Elhadi Adam, ${ }^{1,3}$ Elfatih M. Abdel-Rahman, ${ }^{1}$ Onisimo Mutanga \\ ${ }^{1}$ School of Agriculture, Earth and Environmental Sciences, Pietermaritzburg Campus, University of \\ KwaZulu-Natal, Scottsville P/Bag X01, Pietermaritzburg 3209, South Africa \\ ${ }^{2}$ School of Geography, Archaeology and Environmental Studies, University of the Witwatersrand, \\ Private Bag 3, Wits 2050, Johannesburg, South Africa. \\ ${ }^{3}$ International Centre for Insect Physiology and Ecology (ICIPE), P.O. Box 30772, 00100 Nairobi, \\ Kenya
}

DOI: http://dx.doi.org/10.4314/sajg.v7i1.1

\begin{abstract}
Maize is one of the most important subsistence and commercial crops in the world. In Africa, it is regarded as one of the most popular food crops. Recently however, significant losses due to Phaeosphaeria leaf spot (PLS) infestation have been reported. Therefore, techniques for early detection of PLS infestation are valuable for mitigating maize yield losses. Recently, remotely sensed datasets have become valuable in crop assessment. In this study, we sought to detect early PLS infestation by comparing the performance of commonly used higher spatial resolution sensors (WorldView, Quickbird, Sentinel series 2, RapidEye and SPOT 6) based on their spectrally resampled field spectra. Canopy training spectra were collected on leaves with signs of early infestation and healthy leaves spectral characteristics used for comparison. Training data was collected in 2013 growing season while test data was collected under similar conditions in 2014. The Random Forest algorithm was used to establish the Kappa and overall, user and producer's accuracies. Results showed that the RapidEye sensor with an overall classification accuracy of $86.96 \%$ and Kappa value of 0.76 performed better than the rest of the sensors while the Red, Yellow and Red-Edge bands were most useful for detecting early PLS infestation. The value of the RapidEye sensor in detecting early PLS infestation can be attributed to the optimally centred Red Red-Edge bands sensitive to changes in chlorophyll content, a consequent of PLS infestation on maize leaves. The study provides valuable insight on the value of existing sensors, based on their sensor characteristics in detecting early PLS infestation.
\end{abstract}

Keywords: Phaeosphaeria leaf spot, Remote Sensing, sensors Random Forest, Variable importance

\section{Introduction}

Globally, maize is regarded as one of the most important subsistence and commercial crops. In Africa, a number of authors (eg Derera et al. 2007, Sibiya et al. 2011 and Benhin 2008) note that maize remains the most important food crop. However, recently maize production in the tropical and sub-tropical regions of the continent has significantly declined due to the Phaeosphaeria leaf spot 
(PLS) disease infestation (Gonçalves et al., 2013; Moreira et al. 2009). The disease affects maize foliar and is caused by the ascomycete fungus Phaeospharia maydis (Henn.). Initially spotted in India, studies show that it has spread to North and South America, and recently east and southern Africa (Gonçalves et al., 2013; Moreira et al., 2009; Carson, 2005; Sibiya et al., 2011; Derera et al., 2007). The infestation often leads to premature leaf desiccation and consequent reduction in the crop cycle, decrease in grain volume and mass and under severe conditions death of the whole plant (Gonçalves et al., 2013; Paccola et al., 2001). Previous studies have shown that substantial amount (11-60\%) of grain yield is lost in cultivars sensitive to PLS (Derera et al., 2007; Paccola et al., 2001). Whereas losses due to PLS have not been determined in South Africa, agronomic conditions and preferred varieties suggest vulnerability to extensive infestation and possible significant yield losses. Consequently, early detection of infestation and adoption of appropriate mitigation measures is necessary for sustaining subsistence and commercial production.

Traditionally, field visual surveys have been used to determine PLS infestations. This method requires constant monitoring and is often costly, time consuming and impractical on extensive fields (Al-Hiary et al., 2011; Liu, et al., 2008). However, there has been an increased awareness on the value of remotely sensed datasets, particularly crop leaf spectral reflectance characteristics for agricultural applications (Chen et al., 2010; Abdel-Rahman and Ahmed, 2008). Recent advances in sensors on satellite, aerial and ground based platforms in concert with robust algorithms and analysis techniques have further made it possible to reliably determine crop bio-physical status, due to among others change in agronomic conditions and pest and disease infestation (Upadhyay et al., 2012; Pinter et al., 2003). The adoption of remotely sensed datasets in disease mapping has particularly attracted a lot of interest (Estep et al., 2004; Jackson et al., 1986; Baret et al., 2007). Such applications are premised on the fact that disease infestation cause physiological alteration on leaves, hence a change in spectral reflectance (Estep et al., 2004; Jackson et al., 1986; Baret et al., 2007).

Whereas there has been a recent proliferation of "new generation" multispectral sensors (e.g. WorldView-2, Sentinel series 2 and RapidEye) with higher spectral resolution in addition to traditional sensors (e.g. Quickbird and SPOT), choosing the most suitable sensor and bands for early detection of PLS infestation remains a challenge. This is because existing sensors are characterised by a different number of bands and unique spectral configurations, which are sensitive to different vegetation properties. Therefore, in this study, we sought to determine the potential of commonly used and proposed sensors for detecting early PLS infestation. Specifically, we sought to compare the performance of the commonly used multispectral sensors and the importance of each of their respective bands in detecting early PLS infestation by resampling hyperspectral field data.

\section{Materials and methods}

\subsection{Study Area and field spectral data collection}

This study was conducted at the KwaZulu-Natal Department of Agriculture - Cedara Experimental Farm (29³2 S and 30¹6 E), in Pietermaritzburg, KwaZulu-Natal province, South Africa (Figure 1). 


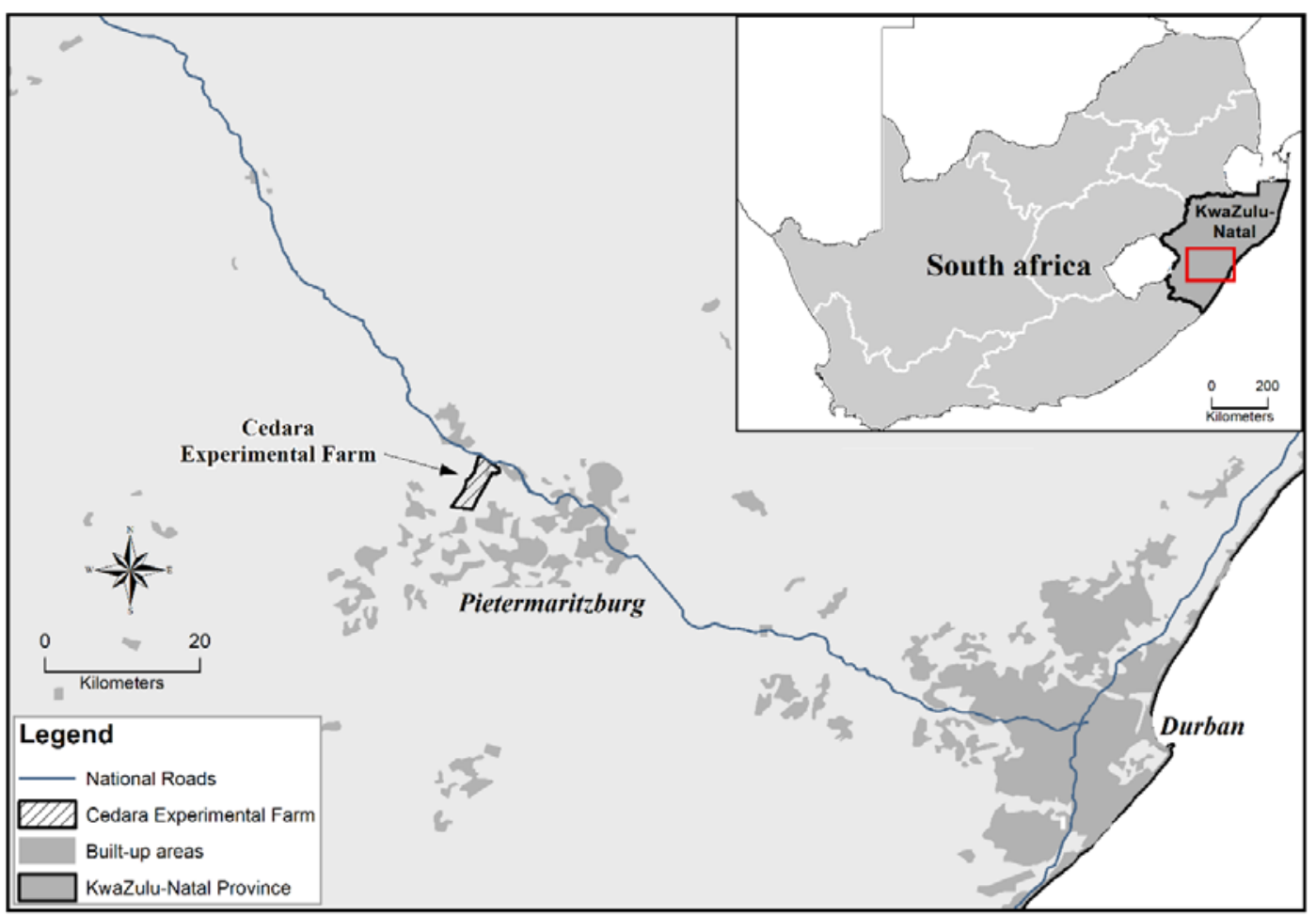

Figure1. The generalised study area showing the Cedara experimental farm.

Field spectral data was collected on $5^{\text {th }}$ of January 2013 and $10^{\text {th }}$ of January 2014 from 138 plots measuring $3 \mathrm{~m}, 0.75 \mathrm{~m}$ and $0.3 \mathrm{~m}$ and $0.75 \mathrm{~m}$ intra row spacing and row length, respectively at full canopy cover. Plots for the study were chosen based on the quantity of leaves with signs of early PLS infestation using optical evaluation (Sibiya et al., 2011). Plots with less than $10 \%$ of the leaves with PLS were regarded as healthy while plots with between 80 to $100 \%$ of the leaves with signs of PLS were regarded to be in the early stage of infestation.

Spectral reflectance for early stage PLS maize canopies were acquired using an Analytical Spectral Device (ASD Inc, Boulder, CO, USA) FieldSpec ${ }^{\circledR} 3$ spectrometer. Spectral data was gathered under sunny and cloud-free conditions between $10 \mathrm{~h} 00 \mathrm{am}$ and $14 \mathrm{~h} 00 \mathrm{pm}$ local time in the two growing seasons ( $5^{\text {th }}$ of January 2013 and $10^{\text {th }}$ of January 2014). The ASD spectral measurement range is 350$2500 \mathrm{~nm}$, measuring radiation at $1.4 \mathrm{~nm}$ intervals for $350-1000 \mathrm{~nm}$ and $2.0 \mathrm{~nm}$ for $1000-2500 \mathrm{~nm}$ spectral regions. Fifteen to twenty measurements were taken from every plot. The Spectral measurements were then averaged to represent the ultimate spectral measurements of each plot. In every 10 to 20 computations, a white based spectral measure was executed on the calibration panel to ameliorate any change in atmospheric conditions and sun irradiance. In total, 66 plots of early infestation and 72 plots of the healthy maize crop were represented separately. Figure 2 provides a visual depiction of healthy leaves, early PLS infestation and respective spectral characteristics. On the $10^{\text {th }}$ of January 2014 (the following growing season) field spectral measurements were acquired in the same location using a similar procedure and comparative conditions. Spectral measurements of 60 and 62 plots for early infestation and healthy maize crop were acquired separately. 

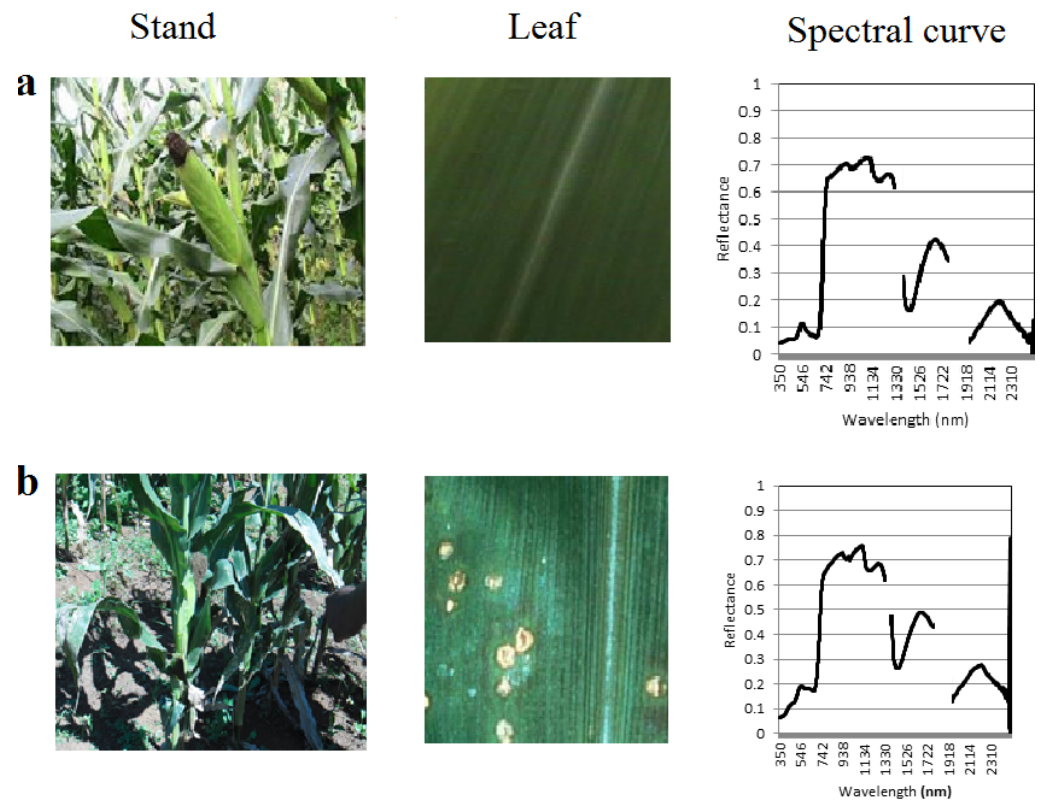

Figure 2. Examples of visual status and spectra of healthy maize (a) and early stage of Phaeosphaeria leaf spot (PLS) infestation (b).

\subsection{Field spectra to sensor resampling}

Spectral reflectance data was resampled to SPOT6, RapidEye, Quickbird, WorldView-2 and Sentinel-2 to band centres using ENVI 4.7 image processing software (Table1). The method used a Gaussian model with a full width at half maximum (FWMAP) equal to the band spacing provided (Mutanga and Skidmore 2005). Spectral reflectance was resampled to the popular multispectral sensors to determine if their respective spectral bands can be used to map the early PLS infestation. If the results are positive, the mapping and monitoring of PLS could then be operational at lower cost using these sensor platforms compared by the use of hyperspectral data.

Table1. Spectral bands and band centres (WorldView-2, Quickbird, Sentinel-2, RapidEye and SPOT 6).

\begin{tabular}{lccc}
\hline Sensor & $\begin{array}{c}\text { Band } \\
\text { description }\end{array}$ & $\begin{array}{c}\text { Spectral } \\
\text { range } \\
\text { (nm) }\end{array}$ & $\begin{array}{c}\text { Band } \\
\text { centre } \\
\text { (nm) }\end{array}$ \\
\hline WorldView-2 & Coastal & $400-450$ & 425 \\
& Blue & $450-510$ & 480 \\
& Green & $510-580$ & 545 \\
& Yellow & $585-625$ & 605 \\
& Red & $630-690$ & 660 \\
& Red-Edge & $705-745$ & 725 \\
& NIR 1 & $770-895$ & 832 \\
& NIR 2 & $860-1040$ & 950 \\
\hline Quickbird & Blue & $450-520$ & 485 \\
& Green & $520-600$ & 560 \\
& Red & $630-690$ & 660 \\
& NIR & $760-900$ & 830 \\
\hline
\end{tabular}




\begin{tabular}{lccc}
\hline Sensor & $\begin{array}{c}\text { Band } \\
\text { description }\end{array}$ & $\begin{array}{c}\text { Spectral } \\
\text { range } \\
\text { (nm) }\end{array}$ & $\begin{array}{c}\text { Band } \\
\text { centre } \\
\text { (nm) }\end{array}$ \\
\hline Sentinel series 2 & Aerosols & $433-533$ & 443 \\
& Classical blue & $458-523$ & 490 \\
& Green & $542-578$ & 560 \\
& Red & $650-680$ & 665 \\
& Red-Edge 1 & $695-713$ & 705 \\
& Red-Edge 2 & $733-748$ & 740 \\
& Red-Edge 3 & $773-793$ & 783 \\
& Red-Edge 4 & $785-900$ & 842 \\
& NIR & $855-875$ & 865 \\
& Water Vapour & $935-955$ & 945 \\
\hline RapidEye & Blue & $440-510$ & 475 \\
& Green & $520-590$ & 555 \\
& Red & $630-685$ & 658 \\
& Red-Edge & $690-730$ & 710 \\
& NIR & $760-850$ & 805 \\
\hline SPOT 6 & Blue & $455-525$ & 490 \\
& Green & $530-590$ & 560 \\
& Red & $625-695$ & 660 \\
& NIR & $760-890$ & 825 \\
\hline
\end{tabular}

\subsection{Statistical analysis}

Random Forest (RF) algorithm was used to classify and predict healthy maize and the early PLS infestation. Random Forest is a bagging process in which various classification trees are created with reference to random subsets of samples arising from training data (Adelabu et al., 2013). The algorithm classifies and measures important variables in high dimensional data such as hyperspectral data and therefore decreases the "curse of dimensionality" without losing important information in the dataset (Adam et al., 2012). For an exhaustive explanation of the Random Forest algorithm see Breiman (2001). In this study, the RF classifications were computed using the 'rattle' package available in R software and used to generate confusion matrices that provide overall accuracy (OA), user accuracy (UA) and producer accuracy (PA). Cohen’s Kappa coefficient were calculated using the formula:

$$
\mathrm{K}=\frac{\operatorname{Pr}(a)-\operatorname{Pr}(e)}{1-\operatorname{Pr}(e)}
$$

Where $\operatorname{Pr}(\mathrm{a})$ is the actual detected agreement and $\operatorname{Pr}(\mathrm{e})$ represents unpredicted agreement (Hallgren, 2012). Kappa gives measured values of agreement between varying observations (Viera and Garrett, 2005). It provides a determination of variability that range from -1 to +1 where +1 represents absolute agreement between different observations, 0 representing an agreement that can happen by coincidence and -1 represent lower possibility of agreement between observations (Viera and Garrett, 2005). 


\subsection{Determination of variable importance}

Variable importance in large datasets identifies a variable that yields outstanding performance compared to other variables. Variable importance in RF computes different measures such as how often a variable is determined, the Gini significance and permutation significance. The permutation importance is considered better than other measures because it assesses value of the variable using mean decrease accuracy based on out-of-bag postulations (Breiman, 2001). The RF can compute the significant variables through the mean decrease in accuracy. Values of mean decrease in accuracy that are high indicate greater significance of that particular variable while low values indicate low significance. Typically, as aforementioned, all bands in the spectrum are focused at a specific range and are sensitive to specific attributes of feature/s being mapped.

\section{Results}

\subsection{Accuracy Assessment}

Table 2 shows the accuracy for Quickbird, RapidEye, Sentinel-2, SPOT 6 and WorldView-2 sensors. The overall accuracy refers to observations that have been correctly classified. User accuracy refers to probability that an observation classified represents a category on the ground while producer accuracy refers to an observation being classified. The overall accuracy values for training data ranged from $83.33 \%$ to $89.13 \%$ while overall values for test data ranged from $79.71 \%$ to $86.96 \%$. Kappa values for training data sets ranged from 0.62 to 0.78 while the accuracy for test data ranged from 0.59 to 0.76 . The Kappa values indicate the level of agreement between the predictions and the classified observations. The highest overall accuracy in the classification of the early stage of PLS was achieved using RapidEye (overall accuracy of $86.96 \%$, producer accuracy of $87.50 \%$, user accuracy of 87.50 and Kappa of 0.76). The lowest overall accuracy on the other hand was obtained using SPOT 6 (overall accuracy of 79.71, producer accuracy of 79.17, user accuracy of 81.43 and Kappa of 0.59 (see Table 2).

Table2. Accuracy Assessment for early stage Phaeosphaeria leaf spot (PLS) infestation using different sensors. User Accuracy (UA), Producer Accuracy (PA), Overall Accuracy (OA). Healthy Stage (HS), Early Stage (ES).

\begin{tabular}{|c|c|c|c|c|c|c|}
\hline \multicolumn{7}{|c|}{ Training data } \\
\hline \multirow[t]{2}{*}{ Sensor } & \multirow[t]{2}{*}{$\mathbf{O A}$} & \multicolumn{2}{|c|}{ UA } & \multicolumn{2}{|c|}{ PA } & \multirow[t]{2}{*}{ KAPPA } \\
\hline & & HS & ES & HS & ES & \\
\hline Quickbird & 83.33 & 84.85 & 81.94 & 81.16 & 85.51 & 0.67 \\
\hline RapidEye & 88.41 & 89.39 & 87.5 & 86.76 & 90 & 0.77 \\
\hline Sentinel Series 2 & 81.16 & 78.79 & 83.33 & 81.25 & 81.08 & 0.62 \\
\hline SPOT 6 & 82.61 & 83.33 & 81.94 & 80.88 & 84.29 & 0.65 \\
\hline WorldView 2 & 89.13 & 90.91 & 87.5 & 86.96 & 91.3 & 0.78 \\
\hline
\end{tabular}




\begin{tabular}{|c|c|c|c|c|c|c|}
\hline \multicolumn{7}{|c|}{ Test data } \\
\hline \multirow[t]{2}{*}{ Sensor } & \multirow[t]{2}{*}{$\mathbf{O A}$} & \multicolumn{2}{|c|}{ UA } & \multicolumn{2}{|c|}{ PA } & \multirow[t]{2}{*}{ КАРPA } \\
\hline & & HS & ES & HS & ES & \\
\hline Quickbird & 82.61 & 81.82 & 83.33 & 81.81 & 83.33 & 0.65 \\
\hline RapidEye & 86.96 & 86.36 & 87.5 & 86.36 & 87.5 & 0.76 \\
\hline Sentinel Series 2 & 80.43 & 75.76 & 84.72 & 81.97 & 79.22 & 0.61 \\
\hline SPOT 6 & 79.71 & 80.3 & 79.17 & 77.94 & 81.43 & 0.59 \\
\hline WorldView 2 & 84.78 & 84.85 & 84.72 & 83.58 & 85.92 & 0.70 \\
\hline
\end{tabular}

\subsection{Variable importance}

Figure 3 shows the performance of sensors rated in terms of variable importance of their respective spectral bands. Based on the mean decrease percentages, the Red band was identified as the most significant in detecting early PLS infestation. Other significant bands include the Yellow and the RedEdge bands.

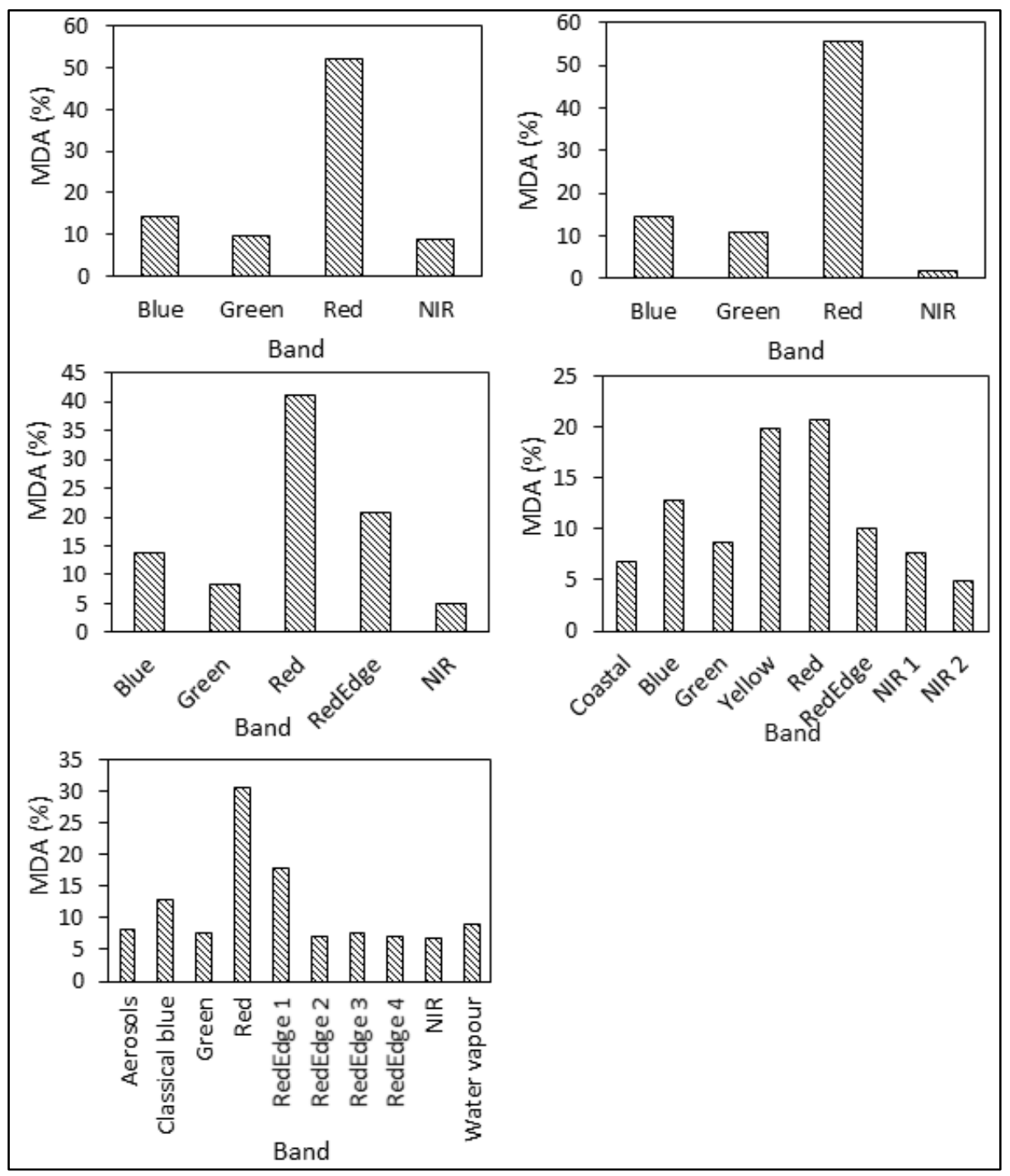

Figure 3. Variable importance of (a) Quickbird, (b) SPOT 6, (c) RapidEye, (d) Worldview-2 and (e) Sentinel-2. (MDA = Mean Decrease in Accuracy). 


\section{Discussion}

This study sought to discriminate between early PLS infestation and the healthy maize crop by resampling hyperspectral data to SPOT 6, Quickbird, RapidEye, Sentinel-2 and WorldView-2. Spectral resampling was performed using the Random Forest, a robust algorithm for predicting and classifying variables. Results in this study show that RapidEye, with an overall accuracy of $86.96 \%$ and Kappa of 0.76, offer better detection of early PLS infestation than other sensors. These findings are consistent with Adam et al. (2012) who found that using relevant bands without redundancy increases classification accuracy in vegetation mapping. According to Adam et al. (2012), the RapiEye's Red and Red-Edge bands are particularly valuable in vegetation analysis. Whereas the WorldView-2 and Sentinel-2 are also characterised by the Red and Red-Edge bands, their overall classification accuracy, 84.78 and 80.43, respectively and were lower in detecting early PLS infestation than the RapidEye.

This study showed that the RF algorithm could be used to determine the value of each of the bands in the commonly used sensors in determining early PLS infestation. Variable importance showed that the Red band in the visible region of the spectrum, the Yellow band and the Red-Edge band were most valuable in detecting early PLS infestation. A number of studies (e.g. Digital Globe, 2010, Zhang et al., 2005 and Apan et al., 2005) have noted the value of the Red band in vegetation studies. Zhang et al. (2005) for instance noted that the Red band was sensitive to crops infested by diseases while Apan et al. (2005) found that the Red band in the visible region was valuable in predicting Alternaria solani fungal infection on tomatoes. The Yellow band's ability to determine early PLS infestation can be attributed to its ability to identify "yellowness", a common characteristic in diseased plants (Digital Globe, 2010; Oliveira et al., 2010 ). The Red-Edge band has the ability to assess plant nutrition, health and discriminate vegetation species (Filella and Peñuelas, 1994; Pinar and Curran, 1996; Daughtry et al., 2000; Rodriguez et al., 2006). According to Eitel et al. (2011), the Red-Edge band can determine both the amount of chlorophyll and Nitrogen, the main determinants of the crop health.

Results obtained from this study demonstrate the potential of multispectral resolution imagery, such as RapidEye in early detection of PLS infestation. The WorldView-2 is also recommended for PLS detection for its three important bands (Red, Yellow and Red-Edge). This study provides an indication of the most useful sensors for PLS infestation. However, for image applications, this must results should be treated with caution as spatial resolution is also known to significantly influence mapping accuracy. The influence of spatial resolution on the optimum sensor's (eg RapidEy) mapping accuracy needs further investigation.

\section{Conclusion}

The aim of this study was to detect early PLS infestation by resampling spectral data to different multispectral sensors. Results in this study showed that early PLS infestation can be better detected using RapidEye's multispectral bands. The significant bands for early PLS infestation were the Red, Yellow, and the Red-Edge bands. Using the Random Forest algorithm, an overall accuracy of 86.96\% 
on RapidEye image to detect early PLS infestation was achieved. This study demonstrates the potential of the Random Forest algorithm in determining the useful sensor and sensor characteristics for early detection of PLS infestation, critical for image up-scales and timely adoption of mitigation measures.

\section{References}

Abdel-Rahman, E. M. \& F. B. Ahmed 2008, 'The application of remote sensing techniques to sugarcane (Saccharum spp. hybrid) production: a review of the literature', International Journal of Remote Sensing, vol. 29, pp. 3753-3767.

Adam, E. M., O. Mutanga, D. Rugege \& R. Ismail 2012, 'Discriminating the papyrus vegetation (Cyperus papyrus L.) and its co-existent species using random forest and hyperspectral data resampled to HYMAP'. International Journal of Remote Sensing, vol. 33, pp. 552-569.

Adelabu, S., O. Mutanga, E. Adam \& M. A. Cho 2013, 'Exploiting machine learning algorithms for tree species classification in a semiarid woodland using RapidEye image', Journal of Applied Remote Sensing, vol. 7, pp. 073480-073480.

Adjorlolo, C., O. Mutanga, M. A. Cho \& R. Ismail 2013, 'Spectral resampling based on user-defined inter-band correlation filter: C3 and C4 grass species classification', International Journal of Applied Earth Observation and Geoinformation, vol. 21 pp. 535-544.

Al-Hiary, H., S. Bani-Ahmad, M. Reyalat, M. Braik \& Z. ALRahamneh 2011, 'Fast and accurate detection and classification of plant Diseases' International Journal of Computer Applications, vol. 17, pp. 31-38.

Apan, A., B. Datt \& R. Kelly 2005. Detection of pests and diseases in vegetable crops using hyperspectral sensing: a comparison of reflectance data for different sets of symptoms. In Proceedings of the 2005 Spatial Sciences Institute Biennial Conference 2005: Spatial Intelligence, Innovation and Praxis (SSC2005), 10-18. Spatial Sciences Institute.

Baret, F., V. Houles \& M. Guèrif 2007, 'Quantification of plant stress using remote sensing observations and crop models: the case of nitrogen management', Journal of Experimental Botany, vol. 58, pp. 869-880.

Benhin, J. K. A. 2008, 'South African crop farming and climate change: An economic assessment of impacts', Global Environmental Change, vol. 18, pp. 666-678.

Breiman, L. 2001, 'Random Forests', Machine Learning, vol. 45 no. 1, pp. 5-32.

Carson, M. 2005, 'Yield loss potential of Phaeosphaeria leaf spot of maize caused by Phaeosphaeria maydis in the United States' Plant disease, vol. 89, pp. 986-988.

Chen, P., D. Haboudane, N. Tremblay, J. Wang, P. Vigneault \& B. Li 2010, ' New spectral indicator assessing the efficiency of crop nitrogen treatment in corn and wheat' Remote Sensing of Environment, vol. 114, pp. 1987-1997.

Daughtry, C.S.T., C.L. Walthall,; M.S. Kim, E. Brown \& J.E. McMurtrey 2000, 'Estimating Corn Leaf Chlorophyll Concentration from Leaf and Canopy Reflectance', Remote Sensing of Environment, vol. 74, no 2, pp. 229- 239.

Derera, J., P. Tongoona, B. Vivek, N. van Rij \& M. Laing, 2007, 'Gene action determining Phaeosphaeria leaf spot disease resistance in experimental maize hybrids', South African Journal of Plant and Soil, vol. 24, pp. 138.

Digital Globe 2010, The Benefits of the 8 Spectral Bands of WorldView-2.White Paper. Digital Globe ${ }^{\circledR}$, USA. 
Eitel, J.U.H., L.A. Vierling, M.E. Litvak, D.S. Long, U. Schulthess, A.A. Ager, D.J. Krofcheck \& L. Stoscheck 2011, 'Broadband, red-edge information from satellites improves early stress detection in a New Mexico conifer woodland', Remote Sensing of Environment, vol. 115, pp. 3640-3646.

Estep, L., G. Terrie \& B. Davis 2004, 'Crop stress detection using AVIRIS hyperspectral imagery and artificial neural networks', International Journal of Remote Sensing, vol. 25, pp. 4999-5004.

Filella, I. \& J. Peñuelas 1994, 'The red edge position and shape as indicators of plant chlorophyll content, biomass and hydric status', International Journal of Remote Sensing, vol. 15, S. pp. 1459-1470.

Gonçalves, R., J. Figueiredo, E. Pedro, W. Meirelles, R. L. Junior, A. Sauer \& L. Paccola 2013, 'Etiology of Phaeosphaeria leaf spot disease of maize', Journal of Plant Pathology, vol. 95, pp. 559-569.

Hallgren, K. A. 2012, 'Computing inter-rater reliability for observational data: an overview and tutorial', Tutorials in Quantitative Methods for Psychology, vol. 8, pp. 23.

Jackson, R. D., J. R. Pinter, R. J. Reginato \& B. I. Sherwood 1986, 'Detetction and evaluation of plant stresses for crop management decisions', IEEE Transactions on Geoscience and Remote Sensing, vol. 24, pp. 99-105.

Liu, Z., J. Huang \& R. Tao 2008, 'Characterizing and Estimating Fungal Disease Severity of Rice Brown Spot with Hyperspectral Reflectance Data', Rice Science, vol. 15, pp. 232-242.

Moreira, J., D. Bento, A. Souza \& C. Souza, Jr. 2009, 'QTL mapping for reaction to Phaeosphaeria leaf spot in a tropical maize population', Theoretical and Applied Genetics, vol. 119, pp. 1361-1369.

Oliveira, E. D., F. T. Fernandes, E. Carvalho \& J. D. C. Machado 2010, 'The phaeosphaeria leaf spot of maize in brazil: evidences of a new ethiologic agent', Revista Brasileira de Milho e Sorgo, vol. 3.

Paccola, L., A. Ferreira, W. Meirelles, I. Marriel \& C. Casela 2001, 'Detection of a bacterium associated with a leaf spot disease of maize in Brazil', Journal of Phytopathology, vol. 149, pp. 275-279.

Pinar, A. \& P. J. Curran 1996, 'Grass chlorophyll and the reflectance red edge', International Journal of Remote Sensing, vol. 17, S. pp. 351-357.

Pinter, P. J., J. L. Hatfield, J. S. Schepers, E. M. Barnes, M. S. Moran, C. S. T. Daughtry \& D. R. Upchurch 2003, 'Remote sensing for crop management' Photogrammetric engineering and remote sensing, vol. 69, pp. 647-664.

Rodriguez, D., G.J. Fitzgerald, R. Belford \& L. K. Christensen 2006, 'Detection of nitrogen deficiency in wheat from spectral reflectance indices and basic crop eco-physiological concepts', Australian Journal of Agricultural Research, vol. 57, pp. 781-789.

Sibiya, J., P. Tongoona, J. Derera, N. van Rij \& I. Makanda 2011, 'Combining ability analysis for Phaeosphaeria leaf spot resistance and grain yield in tropical advanced maize inbred lines' Field Crops Research, vol. 120, pp. 86-93.

Upadhyay, P., A. Kumar, P. S. Roy, S. K. Ghosh \& I. Gilbert 2012, 'Effect on specific crop mapping using WorldView-2 multispectral add-on bands: soft classification approach. Journal of Applied Remote Sensing, pp. 6, vol. 063524-1-063524-13.

Viera, A. J. \& J. M. Garrett, 2005, 'Understanding interobserver agreement: the kappa statistic' Family Medicine,vol. 37, pp. 360-363.

Zhang, P., B. Anderson, B. Tan, D. Huang \& R. Myneni, 2005, 'Potential monitoring of crop production using a satellite-based Climate-Variability Impact Index' Agricultural and Forest Meteorology, vol. 132, pp. 344-358. 